\title{
The CAnadian High-Resolution Traumatic Brain Injury (CAHR-TBI) Research Collaborative
}

\author{
Francis Bernard, Clare Gallagher, Donald Griesdale, Andreas Kramer, \\ Mypinder Sekhon, Frederick A. Zeiler (D)
}

\begin{abstract}
In traumatic brain injury (TBI), future integration of multimodal monitoring of cerebral physiology and high-frequency signal processing techniques, with advanced neuroimaging, proteomic and genomic analysis, provides an opportunity to explore the molecular pathways involved in various aspects of cerebral physiologic dysfunction in vivo. The main issue with early and rapid discovery in this field of personalized medicine is the expertise and complexity of data involved. This brief communication highlights the CAnadian High-Resolution Traumatic Brain Injury (CAHR-TBI) Research Collaborative, which has been formed from centers with specific expertise in the area of high-frequency physiologic monitoring/processing, and outlines its objectives.

RÉSUMÉ : Présentation des objectifs du CAnadian High-Resolution Traumatic Brain Injury (CAHR-TBI) Research Collaborative. L'intégration à venir des techniques de suivi multimodal de la physiologie cérébrale et de traitement de signaux à haute fréquence avec les modalités avancées d'analyse de la génomique, de la protéomique et de la neuro-imagerie offre la possibilité d'explorer les voies moléculaires (molecular pathways) impliquées dans divers aspects liés au dysfonctionnement physiologique cérébral in vivo pour des cas de traumatisme cranio-cérébral (TCC). Dans ce domaine personnalisé de la médecine, où les découvertes se font à un stade précoce et de façon accélérée, le principal enjeu demeure le degré de raffinement et de complexité des données concernées. Cette brève présentation veut donc mettre en relief le CAnadian High Resolution Traumatic Brain Injury (CAHR-TBI) Research Collaborative et exposer ses objectifs. Rappelons qu'il s'agit d'un réseau constitué de centres de recherche dotés d'une expertise particulière dans le domaine du suivi physiologique à hautes fréquences et du traitement des données ainsi obtenues.
\end{abstract}

Keywords: Brain injury, Traumatic, Physiology

doi:10.1017/cjn.2020.54

Can J Neurol Sci. 2020; 47: 551-556

Traumatic brain injury (TBI) is one of the leading causes of death and disability within Canada and globally, with more than 50 million people per year suffering a TBI worldwide, incurring costs of nearly $\$ 540$ billion CAD annually across the globe. ${ }^{1}$ Overall, there has been little change in mortality and morbidity associated with TBI in the past four decades of TBI care. This morbidity and mortality are associated with both primary and secondary injury, with primary injury representing the structural damage to the brain at the time of the incident and secondary injury referring to the cascade of systemic and local cellular events that lead to tissue damage within the brain. Little can be done about primary injury, aside from public awareness, policy, and educational campaigns directed at the reduction in head injury incidence. However, the mechanisms involved in secondary brain injury after TBI are amenable to therapeutic intervention, which might lead to improvements in morbidity and mortality.

Over the past twenty years, the acute care for critically ill TBI patients has seen various advances, with the goal of secondary injury reduction. Implementation of guideline-based approaches to the intensive care unit (ICU) has aimed to homogenize TBI care globally ${ }^{2}$ and provide a framework on which to add more advanced monitoring and individualized interventions according to patient-centered physiology and pathophysiology. ${ }^{3}$ Such advances include the application of various multimodal monitoring (MMM) devices, which allow continuous real-time bedside assessments of cerebral physiology. ${ }^{3}$ With the addition and integration of high-frequency signal processing techniques and strategies, one can derive additional continuous cerebral physiologic metrics at the bedside.

From the Section of Critical Care, Department of Medicine, University of Montreal, Montreal, Canada (FB); Section of Neurosurgery, Department of Clinical Neurosciences, University of Calgary, Calgary, Canada (CG, AK); Department of Anesthesiology, Pharmacology \& Therapeutics, University of British Columbia, Vancouver, Canada (DG, MS); Department of Critical Care Medicine, University of Calgary, Calgary, Canada (AK); Section of Neurosurgery, Department of Surgery, Rady Faculty of Health Sciences, University of Manitoba, Winnipeg, Canada (FAZ); Department of Human Anatomy and Cell Science, Rady Faculty of Health Science, University of Manitoba, Winnipeg, Canada (FAZ); Biomedical Engineering, Faculty of Engineering, University of Manitoba, Winnipeg, Canada (FAZ); Department of Medicine, Division of Anaesthesia, University of Cambridge, Cambridge, UK (FAZ) and Centre on Aging, University of Manitoba, Winnipeg, Canada (FAZ)

Received December 17, 2019. Final Revisions Submitted February 10, 2020. Date of Acceptance March 9, 2020.

Correspondence to: Frederick A. Zeiler, Section of Neurosurgery, Rady Faculty of Health Sciences, University of Manitoba, Winnipeg, Canada. Email: Frederick.Zeiler@ umanitoba.ca 


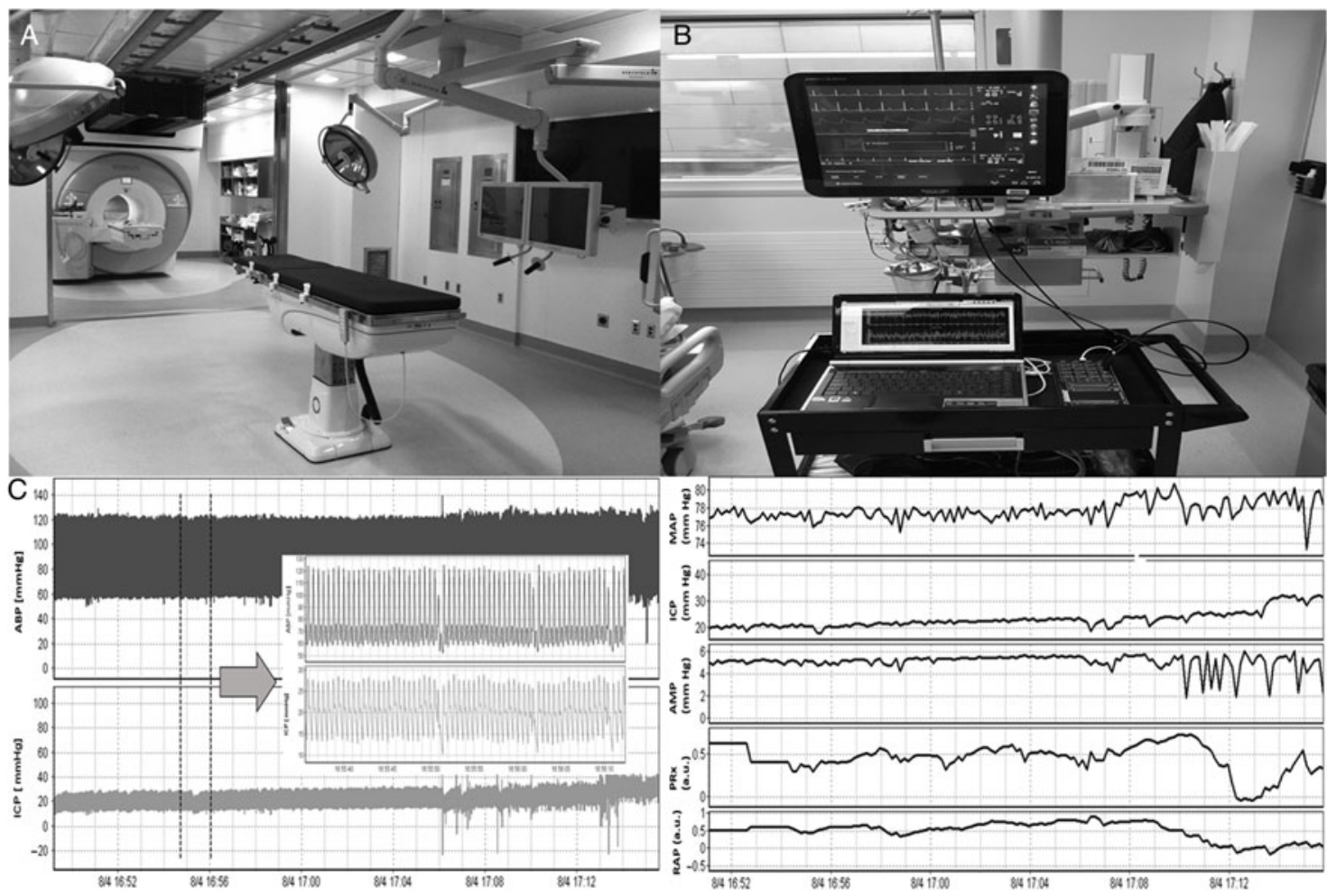

Figure 1: 3 T IMRIS Suite, ICU Bedside Physiologic Recording and Extended Cerebral Physiologic Indices. 3 T $=3$ Tesla, $A B P=$ arterial blood pressure, $A M P=$ pulse amplitude of ICP (derived through Fourier transformed ICP waveform), a.u. $=$ arbitrary units, $C P P=$ cerebral perfusion pressure, $I C P=$ intracranial pressure, $I M R I S=$ intraoperative magnetic resonance suite, $M A P=$ mean arterial pressure, $P R x=$ pressure reactivity index (autoregulation index), $R A P=$ cerebral compensatory reserve index. (A) $3 T$ intraoperative MRI suite located next to intensive care unit. (B) Bedside setup for recording high-frequency digital waveforms of physiologic signals employing intensive care monitoring "plus" (ICM+) data acquisition software (Cambridge Enterprise Ltd, Cambridge, UK, http://icmplus.neurosurg.cam.ac.uk). (C) Left - Recorded digital waveforms of ABP and ICP $(100 \mathrm{~Hz})$, grey arrow displays zoomed window of the full waveforms; Right - processed signals from left window. Signals have been processed to display MAP, AMP, mean ICP, cerebral autoregulation (PRx), and cerebral compensatory reserve (RAP).

Such derived metrics have sparked a new age of monitoring and care for the critically ill TBI patient, ushering in an era where personalized medicine approaches are on the horizon. ${ }^{4}$ Individualized physiologic targets in TBI care, including optimal cerebral perfusion pressure (CPPopt) ${ }^{5,6}$ or individualized intracranial pressure (iICP) thresholds, ${ }^{7}$ are emerging in the literature as viable options at the forefront of personalized medicine in the ICU care of TBI but require more extensive external validation. Similarly, continuous bedside metrics for cerebrovascular reactivity/autoregulation, ${ }^{8}$ compensatory reserve, ${ }^{9}$ autonomics,${ }^{10}$ brain tissue oxygenation $\left(\mathrm{PbtO}_{2}\right),{ }^{11}$ and the cumulative ischemic burden can be employed. ${ }^{12-14}$ These additional measures are of great importance in TBI care, as many have shown to be independent predictors of global outcome in TBI, including cerebrovascular reactivity, CPPopt, and $\mathrm{PbtO}_{2}{ }^{11,15,16}$ Furthermore, derangement in metrics such as cerebrovascular reactivity or $\mathrm{PbtO}_{2}$ are believed to contribute to the relatively unchanged mortality rates in severe TBI over the last $20-30$ years, since the paradigm of care and focus of the past guidelines was on the relatively crude surrogate of cerebral blood flow: absolute ICP measure. ${ }^{17,18}$

Future integration of MMM of cerebral physiology and highfrequency signal processing techniques, with advanced neuroimaging, proteomic, and genomic analysis, provides an opportunity to explore the molecular pathways involved in various aspects of cerebral physiologic dysfunction in vivo. Such work will aim to provide insight into the drivers of impaired cerebral physiology and develop therapeutics aimed at prevention and treatment, with the goal of morbidity and mortality reduction.

Though elegant in concept, the main issue with early and rapid discovery in this field of personalized medicine is the expertise and complexity of data involved. The MMM high-frequency digital physiology requires biomedical engineering expertise for acquisition, storage, and processing. Figure 1 provides an example of such hardware and software integration required for highfrequency signal capture and the need for other advanced capabilities such as integrated neuroimaging. Similarly, coordinating the capture of such valuable physiology with comprehensive information regarding patient-specific demographics and treatment strategies, neuroimaging, and biosamples for future proteomic/genomic analyses requires extensive coordination and collaboration between centers with both interest and expertise in the area. In addition, in order to generate sufficient sample size, such data collection initiates require ongoing prospective collection. Such approaches will also benefit from adaption of artificial intelligence and machine learning techniques.

This brief communication highlights the CAnadian HighResolution Traumatic Brain Injury (CAHR-TBI) Research Collaborative (Figure 2 - CAHR-TBI logo), which has been formed from centers with specific expertise in the area of high-frequency 


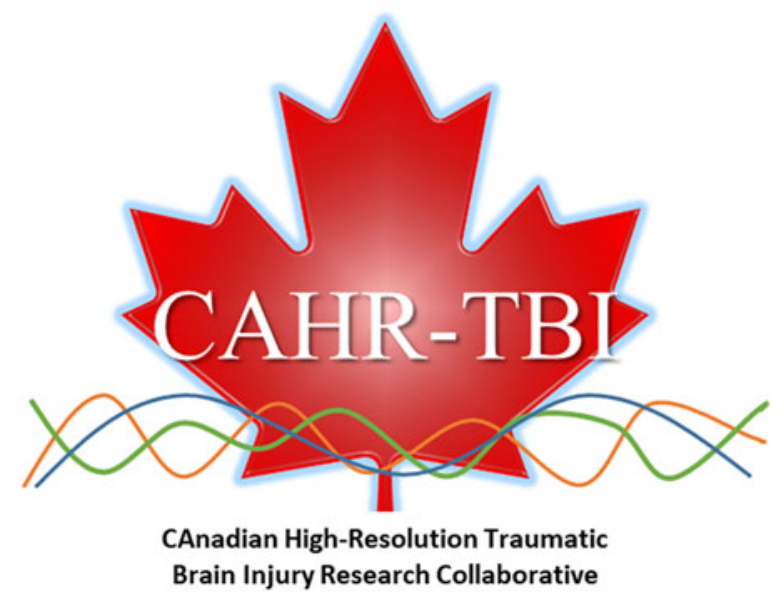

Figure 2: CAnadian High-Resolution Traumatic Brain Injury (CAHR-TBI) Research Collaborative Logo.

physiologic monitoring/processing, consisting of the University of Calgary (AK and CG), University of British Columbia (MS and DG), University of Montreal (FB), and University of Manitoba (FAZ - Location of the Winnipeg Acute TBI Research Program and Winnipeg Acute TBI Biobank (CTRN BRC-00446)). The mandate of this group is to facilitate collaborative highresolution (HR) ICU research for TBI in Canada and become global leaders in this area, with the main goal of understanding drivers of cerebral physiologic dysfunction.

The CAHR-TBI collaborative has the following main objectives:

Facilitate creation of a unique high quality prospectively maintained HR database collaborative within Canada among centers with specialty expertise. Such database elements will include high-frequency digital cerebral physiology from MMM in the ICU, patient characteristics (demographics, injury, treatment, laboratory, imaging, outcomes), and biobank elements (at selected centers).

Enable open data sharing between involved centers to facilitate individual program research interests and provide multidisciplinary support for member-led initiatives.

To engage in Canadian-led collaborative initiatives in MMM for TBI and facilitate multicenter prospective studies into advanced MMM, signal processing, advanced prognostic modeling, big data strategies, and molecular pathways involved in physiologic dysfunction in TBI. Such approaches will also be expanded to other neurologic illnesses, such as aneurysmal subarachnoid hemorrhage and hypoxic ischemic brain injury.

To facilitate training of highly qualified personnel (HQP) in Canada within the field of advanced MMM, physiologic signal processing, and investigation into pathogenesis of secondary injury in TBI, creating the next generation of leaders in the field.

To engage, where appropriate, in national and international initiatives for HR data in TBI, including (but not limited to): Canadian Traumatic Brain Injury Research Consortium, ${ }^{19} \mathrm{Col}-$ laborative European NeuroTrauma Effectiveness Research in Traumatic Brain Injury (CENTER-TBI) study,${ }^{20}$ Genetic Associations In Neurotrauma initiative, in the International Initiative for Traumatic Brian Injury Research (InTBIR), and other regional initiatives (such as those with collaborators in Sweden and Finland).
The collaborative will have a variety of medium- and longterm initiatives, based on individual member interests. We currently house over 300 patients worth of archived high-frequency MMM physiologic data in moderate/severe TBI and expect ongoing prospective yearly collection of $\sim 150$ data sets, some of which will have bio banked blood/cerebrospinal fluid (CSF)/ micodialysate samples for future proteomic/genetic analyses (depending on individual site capabilities). Currently, our areas of focus in high-resolution ICU (HR ICU) data for TBI will include:

A. Validation Studies for Advanced MMM in TBI: Such work will focus on providing multicenter validation to previous single-center retrospective studies in the field. These studies are crucial for such advanced monitoring concepts to be adopted clinically and to facilitate clinical trials on various metrics. Fields of validation include (but are not limited to): cerebrovascular reactivity, CPPopt, compensatory reserve, low-resolution monitoring metrics, newer monitoring indices, individual ICP thresholds, advanced invasive monitoring metrics (such as parenchymal brain tissue oxygen, cerebral blood flow, and microdialysis), and noninvasive cerebral physiology monitoring.

B. MMM Characterization of Cerebral Physiology: Despite a growing literature on MMM-based cerebral physiology, the complex interrelationship is not well understood. Studies on MMM relationships between raw and derived cerebral physiologic metrics will occur to improve our understanding.

C. Development of Noninvasive Modalities for Cerebral Physiologic Monitoring: Such work will focus on developing noninvasive techniques for physiology monitoring, such as cerebrovascular reactivity. This initiative will break ground in development, implementation, and validation of such advanced monitoring during subacute and long-term outcome phases after TBI.

D. Advanced Prognostic Models: Utilizing HR data sets, development of more complex and comprehensive prognostic models will occur. Such initiatives will be facilitated using machine learning and big data strategies. Further, we will integrate regional and provincial community health database infrastructure to link acute HR ICU data sets with socioeconomic outcome variables, so as to better characterize the relationship between acute phase physiologic phenotype and long-term sequelae of moderate/severe TBI.

E. Physiologic "State" Modeling: Knowledge and characterization of MMM cerebral physiologic "state" using timeseries and Bayesian state-space techniques may provide the ability to characterize a patient's individual physiologic state and predict state transitions. Such work will involve engaging machine learning and artificial intelligence to aid with dynamic modeling.

F. Tissue Consequences of Impaired Physiology: Combined HR ICU physiologic data sets with computed tomography (CT) and magnetic resonance imaging (MRI)-based neuroimaging during the acute, subacute, and long-term phases post-TBI will facilitate our understanding of the impact of various physiologic derangements on imagingbased lesion development/progression and tissue fate. Such work will require integration of quantitative imaging 
analysis (via manual or semi-automated lesion segmentation techniques).

G. Uncover Molecular Pathways of Cerebral Physiologic Dysfunction in TBI: Combining HR ICU data sets with biobank whole blood, serum, and cerebrospinal fluid/ microdialysate specimens will enable integration of proteomics and genomics with high-frequency physiology. Such unique data sets are positioned to shed light on molecular pathways involved in cerebral physiologic dysfunction and provide novel targets for prevention and treatment.

H. Chronic Sequelae of TBI: Such initiatives will focus on subacute and long-term characterization of TBI and its impact on cerebral physiology, neuroimaging-based tissue consequences (CT/MRI/positron emission tomography), and clinical phenotype. Works will integrate high-frequency continuous cerebral physiology, neuroimaging techniques, genomics, and serum/CSF proteomics.

\section{Rationale for Creation}

CAHR-TBI was created as a separate and distinct entity from the CTRC. The current chair and lead of the CAHR-TBI initiative has been and currently remains a member of the CENTER-TBI HR ICU substudy. This study has been the largest attempt at a multicenter data collection strategy for high-frequency cerebral physiologic information globally. However, first-hand experience with some of the limitations of large multicenter initiatives, where many centers have varying levels of expertise with such data collection, has sparked the development of CAHR-TBI. The current core members of CAHR-TBI possess expertise in high-frequency (i.e. $100 \mathrm{~Hz}$ or higher) digital physiologic signal capture at the bedside for TBI patients. All members have spent time training with European world leaders in the field, ensuring that data capture across the Canadian sites will be accurate and complete. We are one of only a few groups globally with this type of training and expertise, with the ability to ensure accurate and comprehensive high-frequency data collection going forward. Furthermore, our data acquisition platforms have been harmonized between sites, all utilizing Intensive Care Monitoring "Plus" (ICM+) Cambridge Enterprise Ltd, Cambridge, UK, http://icmplus.neurosurg.cam.ac.uk). All members of CAHR-TBI remain scientist or affiliate members of the CTRC and are fully supportive of its goals and promotion of Canadian TBI research. We fully expect to work closely with them to ensure both of our objectives can be achieved.

\section{Membership}

To date, CAHR-TBI membership consists of only those Canadian centers with expertise and existing hardware/software infrastructure for the bedside capture of high-frequency digital cerebral physiologic signals from MMM. Our current membership consists of centers with individual site leads who have received exposure and training to such specialized data capture at leading European centers. This initial restrictive membership is designed to ensure data integrity and reduce data loss during the initial phases of the CAHR-TBI research collaborative. Future additions to the membership of CAHR-TBI will occur through two streams: (A) Core Membership and (B) Affiliate Membership. All decisions to admit new members occur through direct application to the CAHR-TBI Chair (FAZ) and are reviewed by all current core members for suitability, as outline in the CAHR-TBI Research Collaborative Terms of Reference.

Core membership will be granted based on the individual sites ability to capture and store HR physiology. The CAHR-TBI collaborative is interested in expanding to more centers in Canada, though acknowledge it must be done in a step-wise fashion to ensure consistency in data capture across sites, and that the appropriate training has been obtained. Core members have the ability to request access to and direct specific research initiatives using the data across the CAHR-TBI collaborative.

Affiliate membership will be granted to those interested, based on their involvement or desire for involvement, with individual research initiatives/projects. Such affiliate memberships will extend to those members with additional expertise outside of biomedical signal processing and data capture. Affiliate members will be integral to the multidisciplinary approaches to the complex physiologic and biologic data collected. Interested parties will apply to the CAHR-TBI chair for affiliate membership, with applications reviewed by the core members prior to acceptance.

\section{Data Access}

Access to data collected from the CAHR-TBI collaborative will be granted to core members upon submission of formal application to the chair. Each application will be reviewed by the entire core membership, so to ensure that each participating site agrees with the principles of the proposed study and access to the data. Data will be shared in an entirely deidentified format in line with each local research ethics board approvals. High-frequency physiology information will be sent to the Winnipeg Acute TBI Laboratory, which currently trains doctoral level biomedical engineers and anatomists in such data curation, processing, and analysis. This specific site will ensure homogeneity in signal artifact clearance and processing, led by a site principal investigator with $\mathrm{PhD}$ training in biomedical signal processing and cerebral physiology in TBI. After data processing, linkage with patient demographic, treatment, injury, outcome, and biobank information will occur. Such data sets will be sent to the specific requesting sites for further statistical analysis.

At this point in time, outside requests for data access will not be allowed. Only Core or Affiliate members of the CAHR-TBI collaborative will be allowed to suggest projects and apply for access. Future ability for outside access to data will be discussed among the core membership.

\section{Initial Phase of Studies}

Initially, as we start this venture, we plan for post hoc analysis of existing and ongoing data that is collected. There will be specific prospective studies supported by the group, which will be approved by each core member upon application to the CAHR-TBI collaborative. Much of the initial phase of work will be validation work and ongoing phenotyping of cerebral physiologic dysfunction using MMM in TBI patients. Our current understanding of the landscape of MMM defined cerebral physiologic dysfunction in TBI is poor. Through comprehensive phenotyping, we expect to uncover areas for specific interventional trials, such as targeting individualized physiologic targets, or therapeutics designed to manipulate deranged physiologic parameters. At which point, the CAHR-TBI collaborative will 
shift focus to the support of interventional trials with the help of the CTRC and the Canadian Critical Care Trials Group. The first phase of validation and phenotyping studies using available highfrequency data sets is underway, where we will explore various aspects of cerebral physiology after TBI, including evaluation of critical thresholds of cerebrovascular reactivity, evaluation of dosing/exposures of impaired cerebrovascular physiology and outcome in TBI, evaluation of admission demographic/injury/ treatment characteristics associated with impaired physiology, evaluation of time-series relationships between multimodal cerebral physiology, and evaluation of CPPopt in TBI using different methods of derivation.

\section{Multidisciplinary Expertise}

As outlined in the rational for creation of CAHR-TBI, there exists the need for specialized skill sets to ensure proper data collection and storage, when it comes to high-frequency physiologic signals and integration with other forms of data. All core members of the CAHR-TBI research collaborative have spent time with European leaders in the field of advanced MMM in TBI and are clinical subspecialists in neurotrauma and/or neurocritical care. Our physiologic data collection platforms have been harmonized, employing identical collection systems. Our core groups consist of members with formal graduate training in public health and epidemiology, ensuring expertise in data collection strategies. One of our core members has doctoral research training in cerebral biochemistry and protein biomarkers in TBI, from the global leading center on the topic. Similarly, another member possesses doctoral research training in biomedical signal processing and MMM cerebral physiology in TBI and currently directs an independent laboratory training $\mathrm{PhD}-$ level biomedical engineers and anatomists in such advanced skill sets. Further to this, our members have engaged in various national and international initiatives related to TBI and neurocritical care research in the past, solidifying their experience and understanding of the complexities and limitations of such data collection initiatives.

However, we acknowledge the need for support in our ambitious CAHR-TBI collaborative. Each site engages locally with experts in clinical engineering (for on-site support), biostatisticians (for study-specific support in statistical techniques), and data scientists to aid with database creation and optimization of storage. Further integration of multidisciplinary expertise from biochemistry, genetics, and molecular biology is expected to occur as our data collection expands to include samples for proteomics and genomic analyses. As such, the role for new core and affiliate membership to CAHR-TBI is expected to evolve with the changing needs of the group.

\section{Funding}

Currently, funding for the ongoing prospective collection of patient information and high-frequency digital physiologic cerebral signals is provided by various local, regional, and federal partners, depending on the specific core site. Funding is provided by each site's university through research grants, charitable foundations, existing trauma research funding, and federal grants (such as the National Institutes of Health (NIH) and the Canadian Foundation for Innovation (CFI)). Core members plan for group applications to federal funding opportunities to support ongoing infrastructure and specific prospective studies.

\section{KnOWLedge Translation}

Knowledge translation will occur through the various initiatives from the collaborative. We will generate projects that will be presented locally/nationally/internationally at meetings and publish in peer-reviewed venues regularly. Furthermore, the interests and initiatives will enable sustained training opportunities for HQPs in Canada, attract world-class talent for training, and ensure future growth and productivity for the CAHR-TBI Research Collaborative. All of these aspects will guarantee knowledge translation to various stakeholder parties, including academics, clinicians, and patients, facilitating improvements in patient care for those with TBI in Canada and globally.

\section{Pitfalls in Development}

Creation of such a collaborative is not without its challenges, as highlighted by other Canadian research initiatives that have recently been created..$^{19,21,22}$ Over the prior 6 months, the creation of CAHR-TBI has seen hurdles to overcome. First, each site, and site lead, despite having expertise in this type of high-frequency data collection, all have their own individual areas of interest regarding data usage. The initial phase of developing this group involved extensive written and telephone communications regarding purpose, membership, data access, and objective of the collaborative, so as to ensure all prospective core members approved. This ensured that each individual's research interests could be fostered. Second, drafting formal terms of reference for the CAHR-TBI research collaborative, which went through numerous iterations. This was crucial to ensure that each member's research interest/mandate could be fulfilled, standard data collection and protections were upheld, and that control over data use was clearly outlined prior to the initiation of any studies. Third, funding during the preliminary stages to support ongoing prospective data collection was crucial. Core members had to ensure that each site had available funding and that this model would be sustainable in the long term. Finally, data sharing was a challenge. Each province/region has their own data sharing hurdles to overcome. Deidentified data storage and sharing methodologies eliminated most of these regulatory hurdles for data sharing, with application for post hoc access to each sites database occurring yearly to support ongoing projects.

\section{Conclusions}

We acknowledge that such an undertaking is ambitious. However, it is only through such initiatives that we can hope to shed light on drivers of cerebral physiologic dysfunction in the critically ill TBI patients, uncover mechanisms involved in secondary injury, and develop personalized therapies directed at improving morbidity and mortality. Advancements here will ensure the transition to personalized medicine in acute TBI care.

\section{ACKNOWLEDGMENTS}

FAZ receives research support from the United States NIH through the National Institute of Neurological Disorders and Stroke, the Canadian Institutes for Health Research (CIHR), University of Manitoba Thorlakson Chair in Surgical Research Establishment Fund, the University of Manitoba VPRI Research Investment Fund (RIF), the University of Manitoba Rudy Falk 
Clinician-Scientist Professorship, the Health Sciences Centre Foundation Winnipeg, and the Manitoba Public Insurance (MPI) TBI Research Endowment.

\section{DisClosures}

None.

\section{STATEMENT OF AUTHORSHIP}

$\mathrm{FB}, \mathrm{AK}, \mathrm{CG}, \mathrm{MS}$, and DG were responsible for design, content, and manuscript editing. FAZ was responsible for concept, design, and manuscript composition.

\section{REFERENCES}

1. Maas AIR, Menon DK, Adelson PD, et al. Traumatic brain injury: integrated approaches to improve prevention, clinical care, and research. Lancet Neurol. 2017;16:987-1048.

2. Carney N, Totten AM, O'Reilly C, et al. Guidelines for the management of severe traumatic brain injury, Fourth Edition. Neurosurgery. 2017;80:6-15.

3. Le Roux P, Menon DK, Citerio G, et al. The international multidisciplinary consensus conference on multimodality monitoring in neurocritical care: evidentiary tables: a statement for healthcare professionals from the Neurocritical Care Society and the European Society of Intensive Care Medicine. Neurocrit Care. 2014; 2(21 Suppl):S297-361.

4. Zeiler FA, Thelin EP, Donnelly J, et al. Genetic drivers of cerebral blood flow dysfunction in TBI: a speculative synthesis. Nat Rev Neurol. 2019;15:25-39.

5. Needham E, McFadyen C, Newcombe V, Synnot AJ, Czosnyka $\mathrm{M}$, Menon D. Cerebral perfusion pressure targets individualized to pressure-reactivity index in moderate to severe traumatic brain injury: a systematic review. J Neurotrauma. 2017;34: 963-70.

6. Kramer AH, Couillard PL, Zygun DA, Aries MJ, Gallagher CN. Continuous assessment of "optimal" cerebral perfusion pressure in traumatic brain injury: a cohort study of feasibility, reliability, and relation to outcome. Neurocrit Care. 2019;30:51-61.

7. Zeiler FA, Ercole A, Cabeleira M, et al. Patient-specific ICP epidemiologic thresholds in adult traumatic brain injury: a CENTER-TBI Validation Study. J Neurosurg Anesth. 2019. doi: 10.1097/ANA.0000000000000616.

8. Zeiler FA, Donnelly J, Calviello L, Smielewski P, Menon DK, Czosnyka M. Pressure autoregulation measurement techniques in adult traumatic brain injury, part II: a scoping review of continuous methods. J Neurotrauma. 2017;34:3224-37.
9. Zeiler FA, Kim D-J, Cabeleira M, Calviello L, Smielewski P, Czosnyka M. Impaired cerebral compensatory reserve is associated with admission imaging characteristics of diffuse insult in traumatic brain injury. Acta Neurochir (Wien). 2018;160: 2277-87.

10. Hasen M, Almojuela A, Zeiler FA. Autonomic dysfunction and associations with functional and neurophysiological outcome in moderate/severe traumatic brain injury: a scoping review. J Neurotrauma. 2019;36:1491-504.

11. Okonkwo DO, Shutter LA, Moore C, et al. Brain oxygen optimization in severe traumatic brain injury phase-II: a phase II randomized trial. Crit Care Med. 2017;45:1907-14.

12. Güiza F, Depreitere B, Piper I, et al. Visualizing the pressure and time burden of intracranial hypertension in adult and paediatric traumatic brain injury. Intensive Care Med. 2015;41:1067-76.

13. Güiza F, Meyfroidt G, Piper I, et al. Cerebral perfusion pressure insults and associations with outcome in adult traumatic brain injury. J Neurotrauma. 2017;34:2425-31.

14. Helbok R, Meyfroidt G, Beer R. Intracranial pressure thresholds in severe traumatic brain injury: con : the injured brain is not aware of ICP thresholds! Intensive Care Med. 2018;44:1318-20.

15. Zeiler FA, Ercole A, Cabeleira M, et al. Comparison of performance of different optimal cerebral perfusion pressure parameters for outcome prediction in adult traumatic brain injury: a Collaborative European NeuroTrauma Effectiveness Research in Traumatic Brain Injury (CENTER-TBI) Study. J Neurotrauma. 2019;36:1505-17.

16. Oddo M, Levine JM, Mackenzie L, et al. Brain hypoxia is associated with short-term outcome after severe traumatic brain injury independently of intracranial hypertension and low cerebral perfusion pressure. Neurosurgery. 2011;69:1037-45; discussion 1045.

17. Donnelly J, Czosnyka M, Adams H, et al. Twenty-five years of intracranial pressure monitoring after severe traumatic brain injury: a retrospective, single-center analysis. Neurosurgery. 2019;85:E75-82.

18. Chesnut RM, Temkin N, Carney N, et al. A trial of intracranialpressure monitoring in traumatic brain injury. $\mathrm{N}$ Engl $\mathrm{J}$ Med. 2012;367:2471-81

19. Hutchison JS, Emery C, Gagnon I, et al. The Canadian traumatic brain injury research consortium: epitomizing collaborative research in Canada. J Neurotrauma. 2018;35:1858-63.

20. Maas AIR, Menon DK, Steyerberg EW, et al. Collaborative European NeuroTrauma Effectiveness Research in Traumatic Brain Injury (CENTER-TBI): a prospective longitudinal observational study. Neurosurgery. 2015;76:67-80.

21. Dakson A, Tso MK, Ahmed SU, et al. Launch of the Canadian Neurosurgery Research Collaborative. Can J Neurol Sci. 2017; 44:204-6.

22. Korngut L, Campbell C, Johnston M, et al. The CNDR: collaborating to translate new therapies for Canadians. Can J Neurol Sci. 2013;40:698-704. 